\title{
ALGUNOS CRITERIOS TOPOGRÁFICOS PARA IDENTIFICAR EL ORIGEN ANTRÓPICO DE CÁRCAVAS
}

\author{
F. GALLART \\ Institut de Diagnosi Ambiental i Estudis de 1'Aigua \\ Lluís Solé Sabarís s/n, 08028 Barcelona \\ correo electrónico de contacto: fgallart@idaea.csic.es
}

\begin{abstract}
RESUMEN. En ambientes semiáridos o subhúmedos, existen cárcavas individuales o áreas de cárcavas de origen reciente que pueden haber sido causados por la actividad humana. En algunos casos esta actividad humana no se limita a acciones generalizadas sobre el medio, como la deforestación, sino que en áreas cultivadas se pueden identificar modificaciones artificiales de la red de drenaje, usualmente canales de desagüe de escorrentía o acequias de riego, cuya incisión ha causado el desarrollo de la cárcava. El testimonio oral de estas modificaciones es el primer criterio que nos permite identificarlas pero no es frecuente poder disponer de él. El estudio atento del trazado de las cárcavas y de la red elemental de drenaje puede mostrarnos anomalías que son incompatibles con un desarrollo espontáneo. El trazado anómalo de una cárcava que no sigue el eje de un pequeño valle, que es oblicuo respecto a la pendiente del terreno o que sufre una inflexión no justificada suelen ser los criterios más frecuentes. La identificación de una o varias de estas anomalías puede permitirnos deducir el origen antrópico de redes de cárcavas a las que primariamente atribuiríamos un origen natural por su extensión o encajamiento.
\end{abstract}

ABSTRACT. In semi-arid or sub-humid environments, there are isolate gullies or badland areas that could be caused by human activity. In some cases, particularly in cultivated areas, it is possible to identify artificial disturbances of the drainage network whose incision is the responsible for gully development. A detailed study of the gullies and the basic layout of the drainage can show anomalies that are incompatible with a natural evolution. The anomalous layout of a gully that does not follow the axis of a small valley, the existence of gullies that cross obliquely a hillslope, or a sudden change in the path of the gully use to be the most frequent criteria. The identification of one of several anomalies can allow us to deduce the anthropic origin of badland networks originally attributed to a natural origin. 
Palabras clave: cárcavas, red de drenaje, topografía

Key words: badlands, drainage network, topography

Enviado el 15 de septiembre de 2008

Aceptado el 17 de enero de 2009

\section{Introducción}

En nuestros paisajes mediterráneos nos encontramos frecuentemente con cárcavas (barrancos) o áreas de cárcavas (bad-lands) que, por su elevada actividad, nos hacen sospechar un origen antrópico. En climas secos, estas formas evolucionan muy lentamente y suelen mostrar diversas etapas de evolución, de modo que suelen considerarse anteriores a una actividad humana suficiente para originarlas (Wise et al., 1982; Nogueras et al., 2002). Sin embargo, en climas subhúmedos o húmedos, los ritmos de crecimiento son mucho más rápidos, de modo que su edad puede ser mucho más reciente y su origen antrópico es verosímil (Gallart, 1979; Gallart et al., 2001).

La datación de formas y acumulaciones no es suficiente criterio para asignarles un origen antrópico (algunos ejemplos en Peña et al, 2004). En el presente trabajo se ha partido de la hipótesis de que cualquier forma reciente debe considerarse de origen natural a no ser que se disponga de algún criterio que permita descartar este origen, mostrando el papel directo del hombre.

El objeto de este trabajo es precisamente mostrar algunos ejemplos en los cuales se han podido identificar criterios que permiten descartar un origen natural de cárcavas. El mejor método para utilizar estos criterios es la fotografía aérea esteoroscópica convencional y la verificación en el campo, y para mostrarlos se han utilizado mapas topográficos (originalmente a escala 1:5000) con curvas de nivel de equidistancia de $5 \mathrm{~m}$.

\section{La evidencia directa}

En 1974 me encontraba realizando la cartografía geomorfológica de la Conca d'Òdena (cerca de Igualada, Anoia) que es una depresión erosiva cortada en las margas grises marinas del Eoceno (Masachs, 1950). El objetivo principal era el estudio de los glacis de esta cuenca y sus relaciones con las terrazas del río Anoia y sus afluentes. Sin embargo, la omnipresencia de cárcavas con aspecto muy activo llamó mi atención e inicié algunas observaciones sobre ellas y otras formas actuales (Gallart, 1979; el mapa fue publicado más tarde en Gallart, 1992). Cerca de Cal Bep (Castellolí), decidí peguntar a un vecino si las cárcavas habían sido así toda la vida o si tenía constancia de cambios. Mi interlocutor me mostró una cárcava de unos $20 \mathrm{~m}$ de profundidad que había sido originada por su abuelo al desviar un pequeño torrente hacia 1890 (Fig. 1). El curso natural circulaba por el pequeño valle situado al noreste de Ca l'Aguilera, pero fue desviado mediante un pequeño canal entre Ca l'Aguilera y Cal Bep. Pocos años después se empezó 


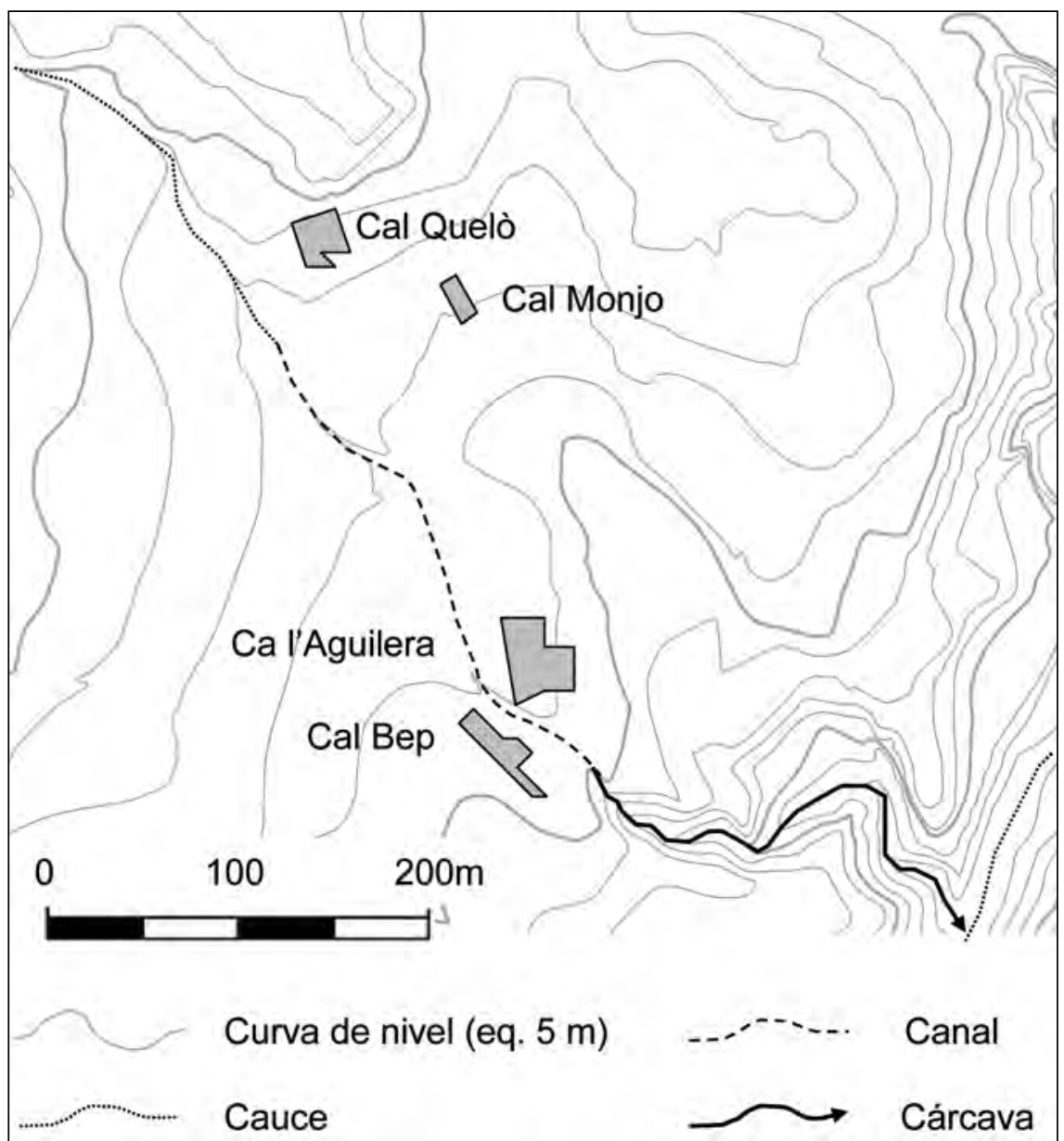

Figura 1. Cárcava formada en las mangas grises del Ecoceno cerca de Castallolí (Anoia) como consecuencia del desvío de un torrente a finales del siglo XIX. El centro del mapa tiene las

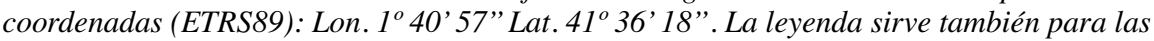
figuras siguientes.

a formar una profunda cárcava encajada en las margas, hasta quedar frenada por unas capas de arenisca en las que están cimentadas estas dos edificaciones.

Lo más relevante de esta información fue que me mostró la importancia de las modificaciones impuestas a la red de drenaje en estas zonas de cultivos en terrazas: existe una red de canales con una densidad muy superior a la de drenaje natural, establecida para evitar la escorrentía sobre los campos de cultivo. Sobre un substrato margoso como el de la Conca d'Òdena, muchos de los cauces principales de esta red se han encajado y forman cárcavas profundas, cuyo trazado evidencia en muchos casos su origen antrópico. Más adelante pude ver que estas modificaciones son mucho más generales de lo que había imaginado al principio, aunque no siempre evolucionan para producir cárcavas (Gallart et al., 1994). 


\section{Cauces desplazados lateralmente en pequeños valles}

Es frecuente observar pequeños valles con fondo plano o en artesa con acumulación coluvial cuyo cauce circula por un margen del valle en lugar de hacerlo por el eje (Fig. 2). Ello permite a los agricultores disponer de campos más grandes con suelos más profundos. En algunos casos la evolución de la cárcava ha sido tan profunda que es difícil reconstruir la disposición de la topografía original antes de la incisión. Las observaciones de campo suelen mostrar pequeños canales que desvían hacia el cauce lateral las aguas de escorrentía del eje del valle.

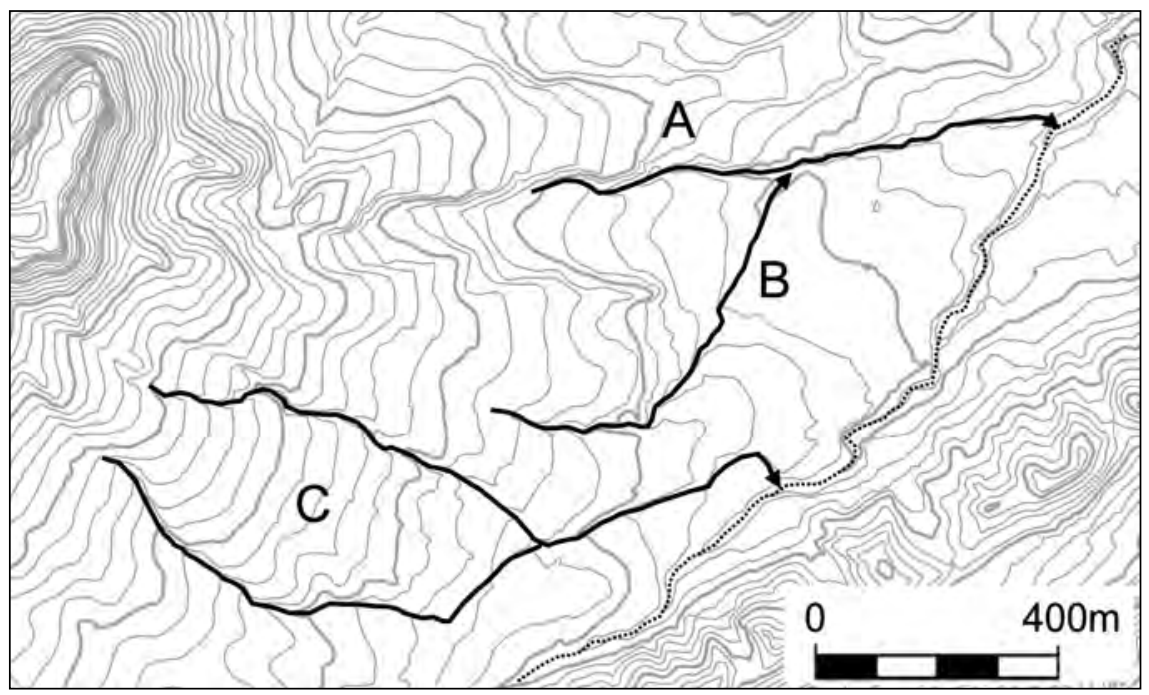

Figura 2. Cárcavas cortadas en glacis pleistocenos y margas grises del Eoceno en los alrededores de Sta. Margarida de Montbui (Anoia). El centro del mapa tiene las coordenadas

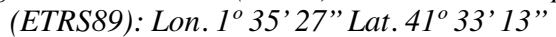

\section{Cauces que no siguen la pendiente máxima de la vertiente}

Otra evidencia de origen antrópico en el trazado de cauces la podemos tener cuando siguen un camino oblicuo a la máxima pendiente de las vertientes. En la Fig. 3 se muestra un ejemplo observado en sedimentos torrenciales Miocenos poco consolidados en La Cerdanya, donde las cárcavas no se relacionan con canales de desagüe sino con acequias de regadío. La cárcava A tiene un trazado 'natural' siguiendo la máxima pendiente de la vertiente. En cambio, la cárcava B tiene un trazado fuertemente oblicuo respecto a la pendiente de la vertiente y solamente recibe afluentes por la izquierda, que siguen la máxima pendiente de la vertiente original. Toda el área está recorrida por una densa red de canales de riego, de modo que lo más verosímil es que la cárcava B fuera originada por brazo de canal que fue construido descendiendo oblicuamente por la vertiente. 


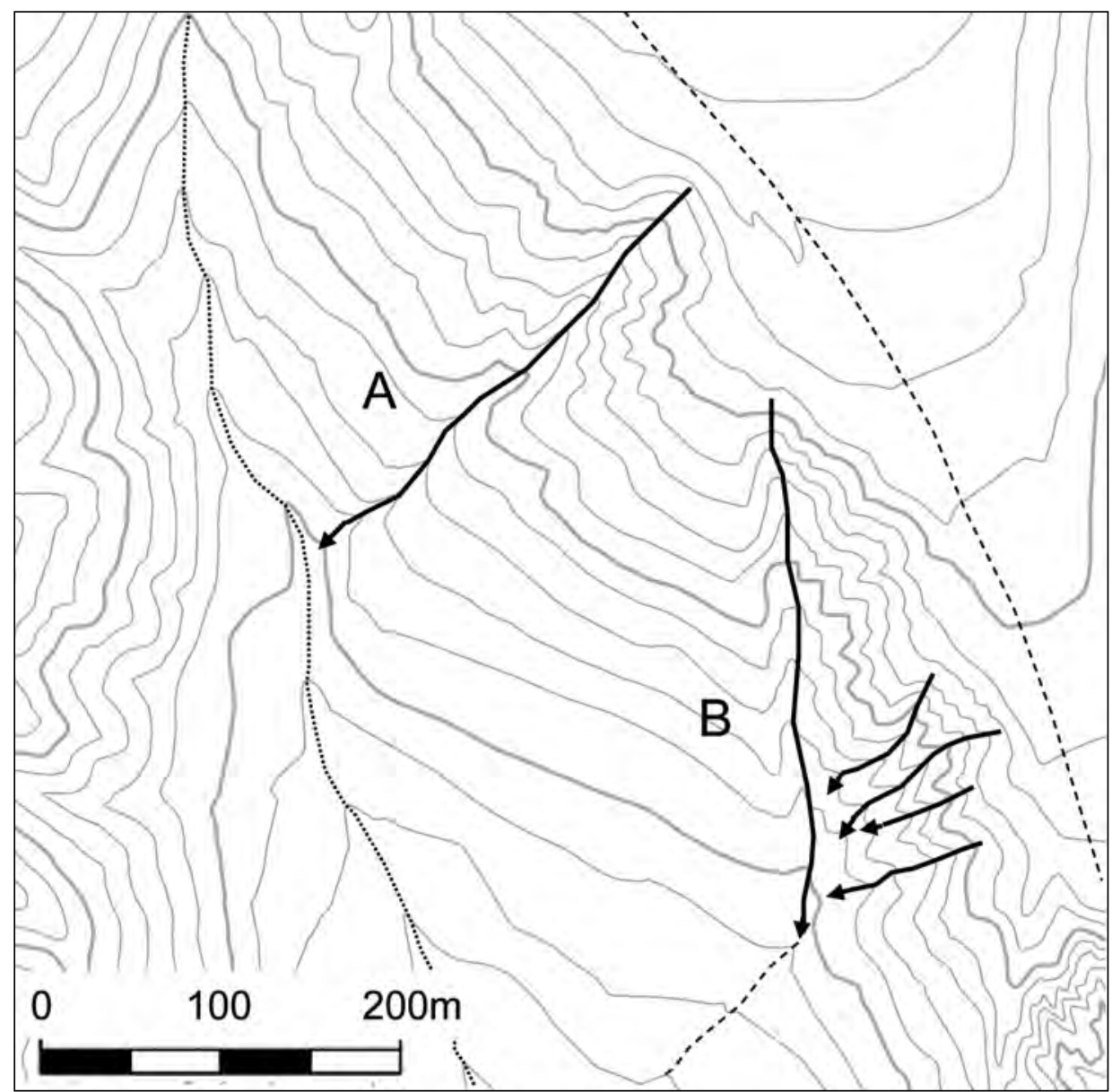

Figura 3. Cárcavas cortadas en una formación torrencial poco consolidada del Mioceno en los alrededores de All (Cerdanya). El centro del mapa tiene las coordenadas (ETRS89): Lon. $1^{\circ} 49^{\prime}$ 27" Lat. $42^{\circ} 23^{\prime}$ 53"

\section{Trazado complejo de redes de cárcavas}

En áreas de pendiente relativamente suave podemos encontrar redes de cárcavas dispersas con trazados complejos en los cuales hay algunas evidencias localizadas de un origen antrópico que nos permiten inferirlo para todo el conjunto. En la misma Fig. 2 pueden verse varios ejemplo de este tipo. Hemos visto que la cárcava A tiene un origen antrópico por su trazado lateral en un pequeño valle. La cárcava B se inicia en la parte baja de un glacis, sin indicios claros de origen artificial, pero luego sufre una inflexión de casi $80^{\circ}$ y atraviesa oblicuamente un glacis de poca pendiente para confluir con la cárcava A. Las dos ramas de la cárcava $\mathrm{C}$ tienen un origen antrópico en cabecera por su 
situación lateral, pero después este criterio se desvanece; sin embargo, ambas ramas sufren después sendas inflexiones de 60 y $80^{\circ}$ respectivamente para terminar con un trazado oblicuo a la pendiente general.

Otro caso similar podemos ver en la Fig. 4: las dos cabeceras de la cárcava indicada tienen un origen artificial, ya que se inician en sendas convexidades laterales de la vertiente, donde el flujo natural tendería a dispersarse en lugar de concentrarse, y la rama derecha recoge las aguas de un pequeño valle cultivado a través de un camino que actúa de canalización. Además, poco después de la confluencia, la cárcava sufre una inflexión de casi $90^{\circ}$ de un modo totalmente injustificado, ya que las curvas de nivel muestran que la vaguada sigue el camino casi rectilíneo. La cárcava acaba con una incisión de más de $15 \mathrm{~m}$ de profundidad de trazado meandriforme, lo que sugiere una evolución dilatada y caudales sostenidos.

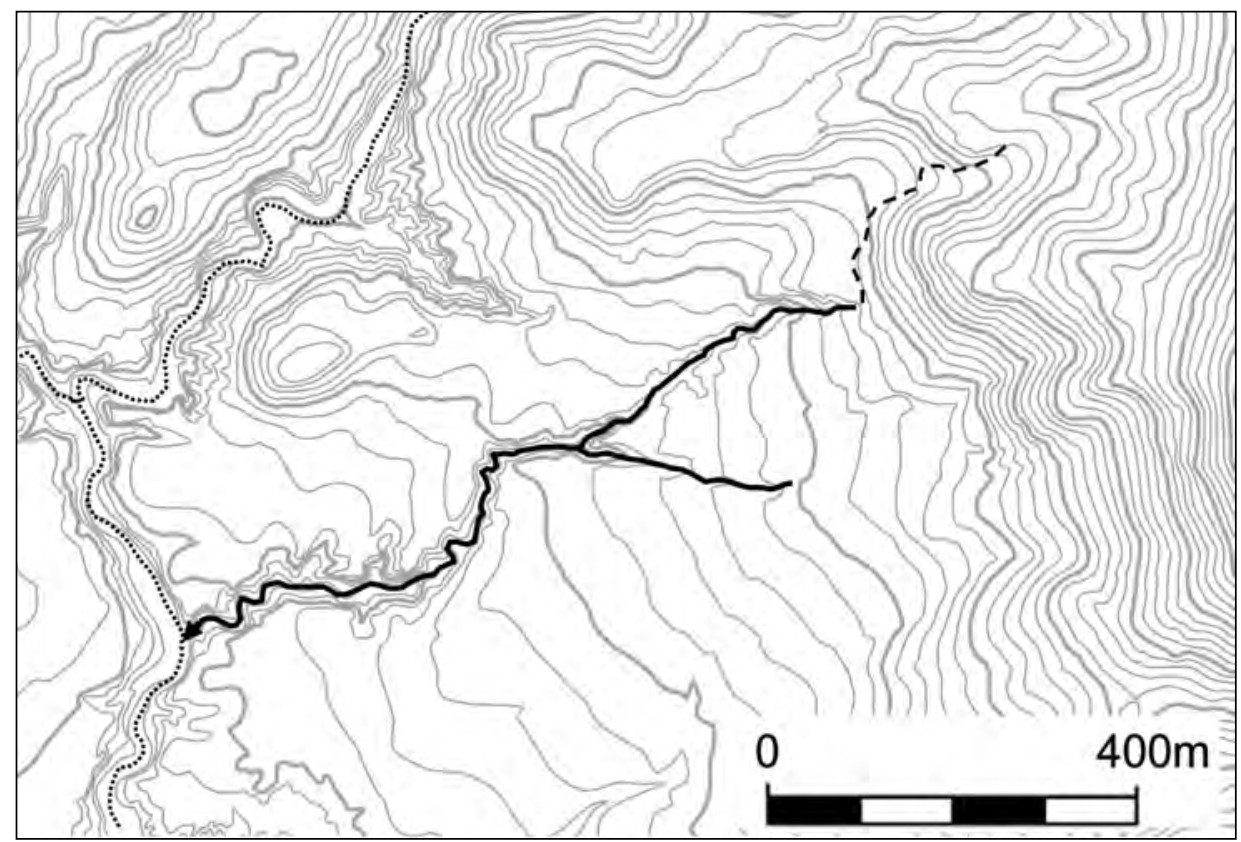

Figura 4. Cárcavas cortadas en glacis pleistocenos y margas grises del Eoceno en los alrededores de Òdena (Anoia). El centro del mapa tiene las coordenadas (ETRS89): Lon. 1'39'44"'Lat. 41 36'17'"

\section{Agradecimientos}

Este trabajo es una contribución al proyecto PROBASE (CGL2006-11619/HID), financiado por el Ministerio de Ciencia e Innovación. El ejemplo de la Fig. 3 ha sido identificado durante los trabajos del proyecto EXCAVA, financiado por la Generalitat de 
Catalunya. Los mapas de curvas de nivel pertenecen a la cobertura 1:5000 del Institut Cartogràfic de Catalunya, propietario del Copyright, y su presentación ha sido posible gracias a la ayuda de Juliana Delgado. Agradezco a José María García Ruiz la invitación a participar en este número extraordinario de Cuadernos de Investigación Geográfica.

\section{Referencias bibliográficas}

GALLART, F. (1979) Observaciones sobre la geomorfología dinámica actual en la Conca d'Òdena (Alrededores de Igualada, provincia de Barcelona). En Actas de la III Reunión del Grupo Español de Trabajo del Cuaternario (Muñoz, J., Aleixandre, T y Gallardo, J., Eds.). CSIC, 123-134 pp., Madrid.

GALlaRT, F. (1992): Estudi Geomorfològic de la Conca d'Odena. L'Estrat, 3: 9-45, Igualada (Barcelona).

GALlART, F., LLORENS, P., LATRON, J. (1994): Studying the role of old agricultural terraces on runoff generation in a Mediterranean small mountainous basin. Journal of Hydrology 159: 291-303.

Gallart, F., Solé, A., Puigdefábregas, J., LÁzAro, R. (2001). Badland systems in the Mediterranean. In: Dryland rivers: processes and management in Mediterranean climates. (Bull, N.L. and Kirkby, M.J., Ed.), Wiley, 299-326 pp., Chichester.

MASACHS AlaVEDRA, V. (1950). Observaciones geomorfológicas en la Conca d'Òdena. Act. y Com. De la $1^{a}$ Asamblea Intercomarcal de Investigadores del Penedès y Conca d'Òdena, Martorell. Igualada.

Nogueras, P., Burjachs, F., Gallart, F., Puigdefàbregas, J. (2000): Recent gully erosion in the El Cautivo badlands (Tabernas, SE Spain). Catena, 40 (2): 203-216.

PeÑA, J.L., Julián, A., ChueCA, J.M EcheverríA, M.T., Ángeles, G.R. (2004). Etapas de evolución Holocena en el valle del río Huerva: Geomorfología y Geoarqueología. En: Geografía Física de Aragón. Aspectos generales y temáticos (Peña, J.L., Longares, L.A. y Sánchez, M., Eds.) Universidad de Zaragoza e Institución Fernando el Católico, 289-302 pp., Zaragoza.

WiSE, S.M., ThORNES, J.B., GILMAN, A. (1982). How old are the badlands? A case study from south-east Spain. In Badland geomorphology and piping (Bryan \& Yair, Eds.) Geo Books, 259-278 pp., Norwich, England. 